\title{
Guaicuru caá, Cienfuegosia drummondii (A. Gray) Lewton, planta medicinal en el Chaco brasileño: conocimiento de la Ruta Bioceánica
}

\author{
Guaicuru caá, Cienfuegosia drummondii (A. Gray) Lewton, planta de uso \\ medicinal no Chaco brasileiro: saberes na Rota Bioceânica \\ Guaicuru caá, Cienfuegosia drummondii (A. Gray) Lewton, medicinal plant in \\ the Brazilian Chaco: knowledge on the Bioceanic Route
}

\author{
Maristela Benites ${ }^{1}$ \\ Simone Mamede ${ }^{2}$ \\ Rosemary Matias ${ }^{3}$ \\ Icléia Albuquerque de Vargas ${ }^{2}$
}

\begin{abstract}
Recibido el 6 de jul. de 2021; revisado e aprobado el 18 de sept. de 2021; aceptado el 13 de oct. de 2021 DOI: http://dx.doi.org/10.20435/inter.v22i4.3447
\end{abstract}

\begin{abstract}
Resumen: Guaicuru caá (Cienfuegosia drummondii) es una especie del Chaco que se usa frecuentemente como planta medicinal en Porto Murtinho, Mato Grosso do Sul, Brasil y en otras regiones del Chaco sudamericano. El objetivo de este trabajo fue realizar el análisis fitoquímico de $C$. drummondii, con el fin de determinar las principales clases de sustancias químicas presentes en el extracto de hoja y describir las principales formas de uso y aplicación medicinal de esta planta por la población de Porto Murtinho, Mato Grosso do Sul, desde la perspectiva de la etnobotánica y del desarrollo local sostenible. La planta fue recolectada en la zona rural del municipio, en octubre de 2017, y los análisis se realizaron en el laboratorio. Las principales clases de metabolismo secundario encontradas en el extracto etanólico de las hojas de $C$. drummondii correspondieron a compuestos fenólicos, flavonoides y antraquinonas, estos últimos con $100 \%$ de frecuencia. Todavía hay una falta de estudios fitoquímicos y farmacológicos de plantas del Chaco brasileño, muchas de las cuales son comúnmente utilizadas como hierbas medicinales por las comunidades locales. Por tanto, la valorización y protagonismo de diferentes prácticas culturales no hegemónicas, como las vinculadas a la etnobotánica y la etnociencia en general, pueden atribuir un valor superlativo al turismo cultural y propuestas de desarrollo en la ansiada Ruta de la Integración Latinoamericana.
\end{abstract}

Palabras clave: etnobotánica; Flora del Chaco brasileño; prospección fitoquímica; sostenibilidad.

Resumo: Guaicuru caá (Cienfuegosia drummondii) é uma espécie chaquenha frequentemente utilizada como planta medicinal em Porto Murtinho, Mato Grosso do Sul, Brasil, e em outras regiões do Chaco sulamericano. O objetivo deste trabalho foi proceder à análise fitoquímica $C$. drummondii, a fim de determinar as principais classes de substâncias químicas presentes no extrato foliar e descrever as principais formas de uso e aplicação medicinal desta planta pela população de Porto Murtinho, Mato Grosso do Sul, na perspectiva da etnobotânica e do desenvolvimento local sustentável. A planta foi coletada na zona rural do município, em outubro de 2017 e as análises foram conduzidas em laboratório. As principais classes de metabólicos secundários encontrados no extrato etanólico das folhas de C. drummondii corresponderam a compostos fenólicos, flavonoides e antraquinonas, estes últimos com 100\% de frequência apresentada. Ainda há carência de estudos fitoquímicos e farmacológicos das plantas do Chaco brasileiro, muitas das quais são comumente utilizadas como ervas medicinais pelas comunidades locais. Portanto, a valorização e o protagonismo das diferentes práticas culturais não hegemônicas, como aquelas vinculadas à etnobotânica e à etnociência geral, poderão atribuir valor superlativo ao turismo cultural e às propostas de desenvolvimento na almejada Rota de Integração Latino-Americana.

Palavras-chave: etnobotânica; flora chaquenha brasileira; prospecção fitoquímica; sustentabilidade.

Abstract: Guaicuru caá (Cienfuegosia drummondii) is a Chaco species frequently used as a medicinal plant in Porto Murtinho, Mato Grosso do Sul, Brazil, and in other regions of the South American Chaco. The objective of this work was to carry out the phytochemical analysis of $C$. drummondii, in order to determine the main classes of chemical substances present in the leaf extract and to describe the main forms of use

\footnotetext{
${ }^{1}$ Instituto Mamede de Pesquisa Ambiental e Ecoturismo, Campo Grande, Mato Grosso do Sul, Brasil.

${ }^{2}$ Universidade Federal de Mato Grosso do Sul, Campo Grande, Mato Grosso do Sul, Brasil.

${ }^{3}$ Universidade Anhanguera Uniderp, Campo Grande, Mato Grosso do Sul, Brasil.
} 
and medicinal application of this plant by the population of Porto Murtinho, Mato Grosso do Sul, from the perspective of ethnobotany and sustainable local development. The plant was collected in the rural area of the municipality, in October 2017 and the analyzes were conducted in the laboratory. The main classes of secondary metabolic found in the ethanol extract of the leaves of $C$. drummondii corresponded to phenolic compounds, flavonoids and anthraquinones, the latter with $100 \%$ frequency presented. There is still a lack of phytochemical and pharmacological studies of plants from the Brazilian Chaco, many of which are commonly used as herbal medicines by local communities. Therefore, the valorization and protagonism of different non-hegemonic cultural practices, such as those linked to ethnobotany and general ethnoscience, may attribute a superlative value to cultural tourism and development proposals in the longed for Latino American Integration Route.

Keywords: ethnobotany; Brazilian Chaco flora; phytochemical prospection; sustainability.

\section{INTRODUCCIÓN}

Los estudios que valorizan el conocimiento sobre la biodiversidad son necesarios tanto para comprender la relación establecida entre el ser humano y otros elementos de la naturaleza, como para el desarrollo de la ciencia y las políticas públicas orientadas al uso sostenible de los bienes naturales y la conservación de la sociobiodiversidad.

La etnobotánica se interesa por la relación entre plantas y diferentes grupos sociales humanos, desde la atribución de nombres, hasta las formas de uso, manejo y aplicación (PANDEY; TRIPATH, 2017). Para Leff (2009), la etnobotánica está vinculada a estudios antropológicos de representaciones simbólicas de diferentes pueblos sobre su entorno y sus formas de utilizarlo, cuyo conocimiento resulta de la combinación de procesos ecológicos, culturales e históricos. La farmacología y la fitoquímica, a su vez, se aplican para investigar las propiedades químicas, bioactivas y farmacológicas presentes en varios organismos vivos y su uso potencial en farmacología y biotecnología. La unión con la etnobotánica puede culminar en la bioprospección de productos naturales y resultar en el desarrollo local y la conservación de la flora nativa.

Así, la etnobotánica conecta varias áreas del conocimiento y tiene un carácter trans y multidisciplinario. La relación entre personas y plantas, aliada a la multiplicidad de temas, ciencias y niveles interactivos que la involucran, resulta en un complejo y dinámico sistema de interacciones que permite calificar a la etnobotánica como una ciencia que apunta al progreso humano (ALBUQUERQUE et al., 2017).

El uso de plantas para combatir diferentes enfermedades y tratamientos es antiguo y se estima su establecimiento desde el origen de la especie humana (MACIEL; PINTO; VEIGA-JUNIOR, 2002). Los estudios etnobotánicos han sido útiles tanto para valorar el conocimiento popular y el tradicional (MACIEL; PINTO; VEIGA-JUNIOR, 2002; ALBUQUERQUE et al., 2017) y orientar propuestas para la conservación de la sociobiodiversidad y la sostenibilidad (GUARIM-NETO; MORAIS, 2003; OLIVEIRA et al., 2009).

La integración entre conocimientos tradicionales y científicos puede resultar en beneficios múltiples y recíprocos, generando fortalecimiento y desarrollo de territorios. En este contexto, Leff (2009) señala que el diálogo de saberes abre una posibilidad de entender la realidad desde diferentes lógicas; establece un diálogo intercultural basado en identidades colectivas y sentidos subjetivos, superando la simple integración de objetos de conocimiento fragmentados.

La familia Malvaceae Juss. es ampliamente reconocido por sus usos en la medicina popular, que van desde la aplicación tópica para tratamientos dermatológicos y cicatrización de heridas hasta enfermedades relacionadas con los sistemas digestivo, circulatorio, respiratorio 
y reproductivo (POTT; POTT, 1994; RODRIGUES; CARVALHO, 2001; JESUS et al., 2009; GIRALDI; HANAZAKI, 2010; LIMA; MAGALHÃES; SANTOS, 2011; CORDEIRO; FÉLIX, 2014; MESSIAS et al., 2015; SCARPA; ANCONATANI, 2017).

Cienfuegosia drummondii (A. Gray) Lewton, conocida en la región transfronteriza Brasil/ Paraguay como guaicuru (SALOMÃO et al., 2009), purguita (MOURA, 2016), algodón silvestre, mercurio, guaicuru caá o guaycurú ka'a, tiene hábito herbáceo-arbustivo, con hasta $40 \mathrm{~cm}$ de altura, se presenta en suelos arcillosos, produce flores y frutos de septiembre a mayo, y la corola permanece abierta solo durante la mañana (KRAPOVICKAS, 2003). Habita en zonas con vegetación abierta, sujetas a inundaciones (matorral higrófito), cuyo estrato herbáceo está dominado por poaceae y ciperaceas, donde hay especies como Copernicia alba (Karanday) y Prosopis spp. (MERELES, 2005; VOGT, 2013). Además de Brasil, está presente en el Chaco argentino y paraguayo (KRAPOVICKAS, 2003) con poblaciones disjuntas en el sur de Texas, EE. UU. (FRYXELL, 1967), donde se recolectaron especímenes-tipo (LEWTON, 1910).

La especie pertenece a la tribu Gossypieae Alefeld, que incluye los géneros Cephalohibiscus Ulbr., Cienfuegosia Cav., Gossypioides Skovst. ex J.B. Hutch., Gossypium L., Hampea Schltdl., Kokia Lewton, Lebroimecia Fosberg y Thespesia Sol. ex Correa. (KRAPOVICKAS, 2003), popularmente conocidos como algodón lato sensu, cuyos usos en la medicina popular se aplican a tratamientos del sistema respiratorio, tales como: asma, resfriados, bronquitis; inflamación y depuración sanguínea (MOREIRA et al., 2002; FRANCO; BARROS, 2006; LIMA; MAGALHÃES; SANTOS, 2011; RIBEIRO et al., 2014; MESSIAS et al., 2015), también con importancia económica para el uso de fibra de algodón. El género Cienfuegosia es considerado típicamente chaqueño (KRAPOVICKAS, 2003).

A pesar del uso tradicional del guaicuru caá por las poblaciones de Porto Murtinho, Mato Grosso do Sul y municipios aledaños del Chaco paraguayo y argentino (THOMPSON et al., 1971; ARENAS; AZORERO, 1977; KRAPOVICKAS, 2003; SALOMÃO et al., 2009).; KUMAR; KUMAR; PRAKASH, 2012; MOURA, 2016; SCARPA; ANCONATANI, 2017), existen pocos estudios de interés herbal que demuestren las propiedades farmacológicas y refuercen la aplicación popular de $C$. drummondii. Los pocos trabajos describen su aparición como planta hospedante del escarabajo picudo (Anthonomus grandis), conocido por infestar los cultivos de algodón (JONES, 2001; LANTERI; CONFALONIERI; SCATAGLINI, 2003) y pocos describen su uso medicinal y su aplicación por las comunidades del Chaco (ARENAS; AZORERO, 1977; KRAPOVICKAS, 2003; MARTÍNEZ, 2011; SCARPA; ANCONATANI, 2017), especialmente del Chaco brasileño.

En Brasil, está marcada como una especie de distribución restringida y con datos deficientes para el análisis sobre la amenaza de extinción a nivel nacional, lo que la hace de interés para la investigación y la conservación (MARTINELLI; MORAES, 2013). En Rio Grande do Sul, está clasificado en la categoría de amenaza "en peligro" (SISTEMA DE INFORMAÇÃO SOBRE A BIODIVERSIDADE BRASILEIRA [SIBBr], 2021).

El uso de plantas alimenticias nativas por los habitantes del Chaco en Porto Murtinho fue objeto de un estudio etnobotánico desarrollado por Bortolotto et al. (2019) y muestra una relación importante entre la comunidad y las plantas locales.

A pesar del uso frecuente de guaicuru caá por poblaciones del Chaco brasileño, mantenido incluso en patios traseros (MOURA, 2016), aún no se ha reportado la caracterización de sus constituyentes fitoquímicos que corroboran el uso medicinal de esta planta por poblaciones residentes en el Chaco. La relación entre la población y los elementos de la biodiversidad, como 
las plantas y su uso medicinal popular, puede contribuir al desarrollo local sostenible, incluyendo varios sectores económicos, entre los que se destaca el turismo, cuyo dinamismo e impulso se espera con la implementación de la Ruta Bioceánica. Este corredor logístico involucrará directamente varios territorios sudamericanos entre Brasil, Paraguay, Argentina y Chile.

El objetivo de este trabajo fue determinar las principales clases de sustancias químicas presentes en el extracto de hojas de guaicuru caá ( C. drummondii) y describir las principales formas de uso y aplicación medicinal de esta planta por la población de Porto Murtinho, Mato Grosso do Sul, desde la perspectiva de la etnobotánica y el desarrollo local sostenible en la Ruta Bioceánica.

\section{MATERIAL Y MÉTODOS}

\section{1 Área de estudio}

El área de recolección corresponde a Porto Murtinho, al suroeste de Mato Grosso do Sul, municipio que tiene áreas representativas del Chaco típico en Brasil, en el sector del Chaco húmedo (PRADO, 1993). El clima se clasifica como cálido y seco, con precipitación y temperatura promedio de $970,3 \mathrm{~mm}$ y $25^{\circ} \mathrm{C}$, respectivamente, y la temporada de lluvias abarca el período de noviembre a febrero (CARVALHO; SARTORI, 2014). La estacionalidad está marcada más por la variación de las precipitaciones que por la temperatura. Aunque las altas temperaturas pueden alcanzar los $49^{\circ} \mathrm{C}$, las heladas pueden ocurrir ocasionalmente en el período más frío y seco (FREITAS et al., 2013). En Chaco, el suelo tiende a ser salino, arcilloso y en el período húmedo, la compacidad mantiene baja la capacidad de drenaje y provoca inundaciones (PENNINGTON; PRADO; PENDRY, 2000).

La ocurrencia del Chaco en Brasil afecta marcadamente al municipio de Porto Murtinho, especialmente a su porción occidental. En esta región convergen elementos bióticos del Chaco, Cerrado y bosques estacionales caducifolios y semideciduos (SARTORI, 2012), y las formaciones vegetales, especialmente las ubicadas en zonas bajas, son influenciadas, de orden biótico y/o abiótico, por inundaciones estacionales. de agua del río Paraguay que componen los paisajes (Figura 1). 
Figura 1 - Distribución del dominio Chaco en América del Sur, con énfasis en el área de estudio en Porto Murtinho, Mato Grosso do Sul (MS), Brasil

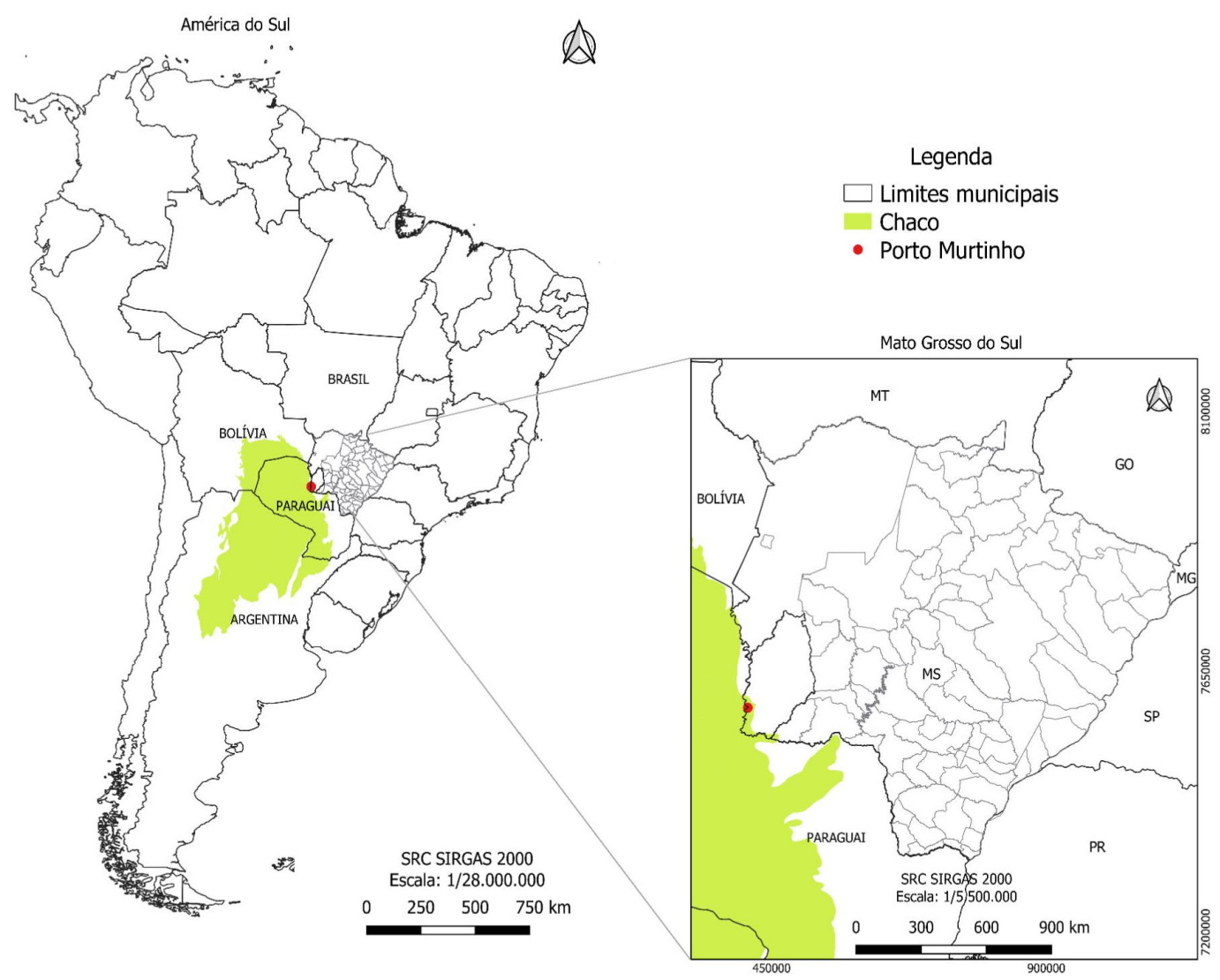

Fuente: Elaboración de las autoras.

Se están llevando a cabo importantes obras de infraestructura en Porto Murtinho, que actualmente se considera un municipio clave para habilitar un corredor de transporte logístico para el flujo de producción y bienes agrícolas entre Brasil, los países adyacentes de América del Sur y los países asiáticos. Este corredor fue acuñado con el término Ruta Bioceánica o Ruta de Integración Latinoamericana.

\subsection{Recolección de datos etnobotánicos sobre guaicuru caá}

Para el relevamiento de información sobre las formas de uso de la planta se utilizaron básicamente dos metodologías: 1) descripción de las formas de preparación y aplicación empleadas por la familia de la autora (MB), cuyos padres nacieron y vivieron en Porto Murtinho, por lo que información recabada de la vida y la historia es en forma oral; 2) diálogos y experiencias no estructuradas con la comunidad de Porto Murtinho para comparar y confirmar formas de uso. También se utilizó un relevamiento bibliográfico para sistematizar los usos y aplicaciones de guaicuru caá por otras comunidades ubicadas en el Chaco Sudamericano, incluyendo Argentina y Paraguay.

\subsection{Recolección de datos botánicos en el campo y preparación de extractos}

Los especímenes recolectados para el análisis fitoquímico provienen de una propiedad

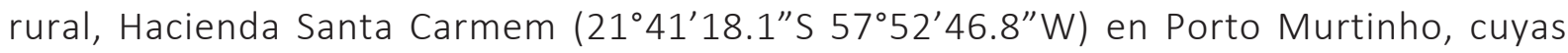


muestras fueron recolectadas en: pastos abandonados, patio residencial y caminos internos de la propiedad.

Las plantas se recolectaron en octubre de 2017, a partir de 10 matrices. Luego de la recolección, se colocaron en bolsas de polietileno en forma de cámara húmeda y se transportaron al Laboratorio de Morfología Vegetal, de la Universidad Uniderp-Anhanguera, donde las muestras fueron herborizadas y secadas para su analisis. Algunos individuos también fueron prensados en el campo para su herborización (Figura 2).

Figura 2 - Guaicuru caá (Cienfuegosia drummondii) y su preparo para herborización, en Porto Murtinho, Mato Grosso do Sul

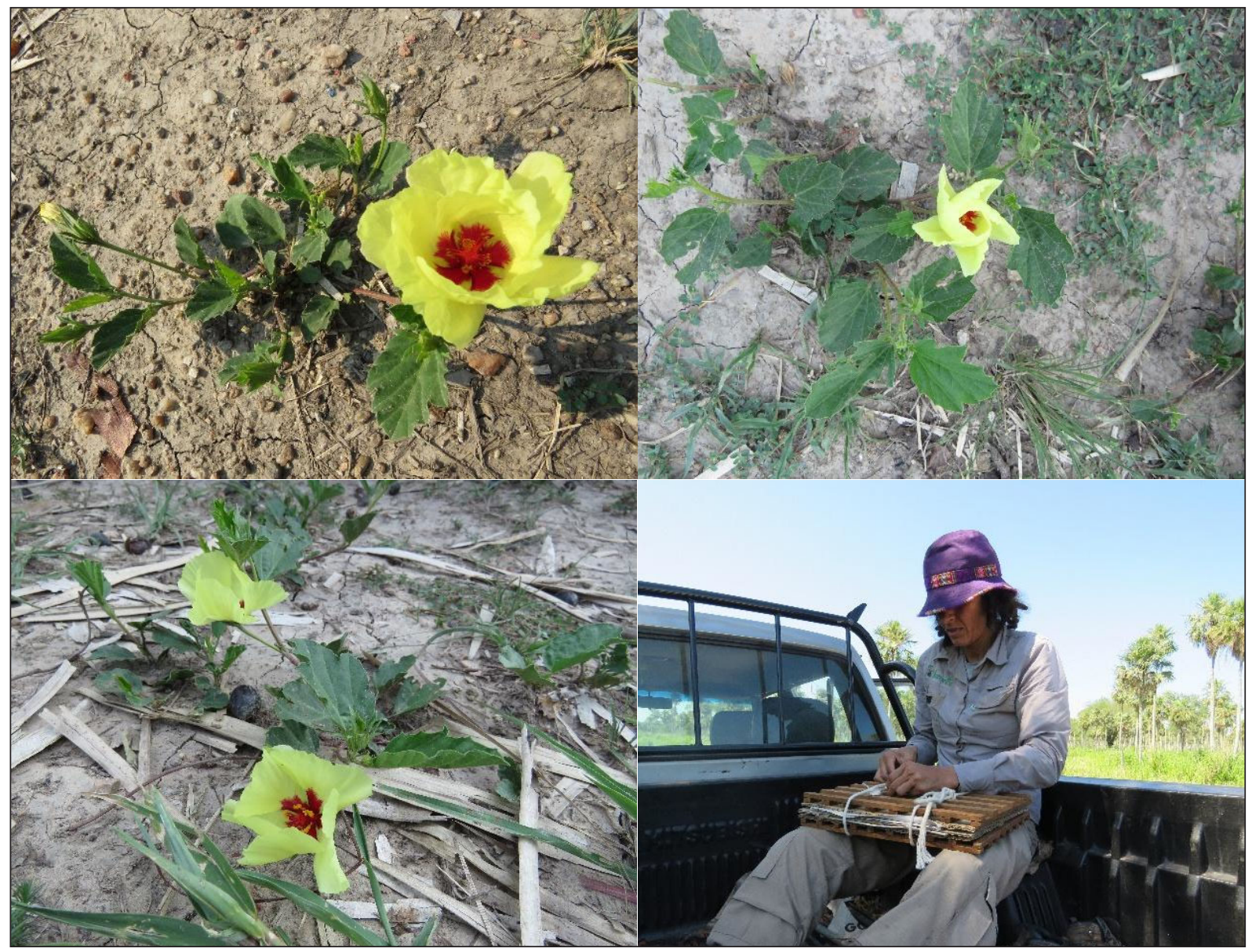

Fuente: Autoras.

\subsection{Extracción y preparación de extracto de etanol crudo de hojas de Cienfuegosia drummondii}

Para la obtención del extracto de etanol crudo, previamente se seleccionaron y pesaron las hojas de la especie en estudio (30g). Después de secar en un horno, pasaron por el proceso de molienda en un molino de cuchillas de acero inoxidable (MARCONI', Modelo MA048) y tamizado. Luego, el material se almacenó en frascos etiquetados y sellados herméticamente, protegidos de la luz y el calor.

La extracción se realizó en un baño de ultrasonidos (UNIDQUE ${ }^{\circ}$, 1450) (60 minutos) y luego se sometió a extracción por maceración estática durante 24 horas. Se filtró el disolvente. Se 
repitió el procedimiento durante diez días y se evaporó el disolvente (rotavapor a $45 \circ \mathrm{C} \pm 5$ 으), y el extracto de etanol crudo se mantuvo en un desecador a presión reducida durante seis horas. El proceso proporcionó $10 \mathrm{~g}$ del extracto etanólico crudo.

\subsection{Prospección fitoquímica de extracto etanólico crudo de hojas de Cienfuegosia drummondii}

Se llevó a cabo una prospección fitoquímica para rastrear las clases de metabolitos secundarios del extracto etanólico de la planta. La metodología se basó en los procedimientos de Matos (2009).

Para leer los resultados se analizó la intensidad del color y/o precipitación, lo cual es indicativo de la alta concentración de uno de los grupos químicos presentes en las especies vegetales. Como estándar, para muestras con color intenso y/o precipitación, se utilizó el extracto etanólico. Todos los tubos eran del mismo tamaño y diámetro; los análisis se realizaron por triplicado y los resultados se expresaron con base en Fontoura et al. (2015) quienes clasifican los cambios de color en: alta intensidad $(+++=100 \%)$, moderada $(++=50 \%)$, baja $(+=25 \%)$, parcial $( \pm=10 \%)$ y negativa $(-=0 \%)$ y ensayos de formación de precipitados (compuestos fenólicos, taninos y azúcares reductores). Se utilizaron tubos de ensayo del mismo tamaño y diámetro y los precipitados de cada análisis se midieron y clasificaron en: alta intensidad $(0,7-1 \mathrm{~cm},+++=$ $100 \%)$, moderada $(0,5-0,7 \mathrm{~cm},++=50 \%)$, baja $(0,2-0,5 \mathrm{~cm},+=25 \%)$, parcial $(<0,2 \mathrm{~cm}, \pm=10 \%)$, negativa sin formación de precipitados ni turbidez.

Parte del extracto etanólico se evaporó y se disolvió en metanol (HPLC) (10 mg mL ${ }^{-1}$ ) y se usó en secuencia para obtener los espectros UV-visible, determinados en el rango de longitud de onda de 200 a $800 \mathrm{~nm}$. El espectro analizado se basó en la literatura técnica (WORKMANJUNIOR, 2001).

\subsection{Medición de compuestos fenólicos, flavonoides y alcaloides}

El contenido total de fenol (TF) se determinó por el Método de Folin-Ciocalteu, con absorbancias medidas en un espectrofotómetro en la región de $750 \mathrm{~nm}$, en cubetas de cuarzo. El análisis se realizó interpolando la absorbancia de las muestras frente a una curva de calibración $\left(y=0,0288 x+0,0098 ; R^{2}=0,9897\right)$, construida con estándares de ácido gálico (GAE 10 a 300 $\mu \mathrm{fg} \mathrm{mL}^{-1}$ ) (SOUSA et al., 2007).

Para cuantificar los flavonoides, se asumió como estándar la quercetina ( $\mathrm{QE}=0,5 \mathrm{mg} \mathrm{mL}^{-1}$ ) para construir la curva de calibración a concentraciones de 0,$04 ; 0,2 ; 0,4 ; 2 ; 4 ; 8 ; 12 ; 16$; y $20 \mu \mathrm{g}$ $\mathrm{mL}^{-1}\left(\mathrm{y}=0,0255 \mathrm{x}+0,1127 \mathrm{R}^{2}=0,9988\right)$. Los análisis se realizaron mediante espectrofotometría a una longitud de onda de $420 \mathrm{~nm}$, en cubetas de cuarzo (PEIXOTO-SOBRINHO et al., 2008).

\section{RESULTADOS Y DISCUSIÓN}

Las principales clases de metabolitos secundarios encontrados en las hojas de $C$. drummondii correspondieron a compuestos fenólicos, flavonoides y antraquinonas, estos últimos con $100 \%$ de frecuencia presentados. La quimiotaxonomía de la familia Malvaceae es diversa y entre las clases de metabolitos secundarios frecuentes se encuentran los flavonoides, terpenoides y ácidos grasos insaturados (SCHMID; PATTERSON, 1988). Los flavonoides y terpenoides también 
se evidenciaron en los resultados del cribado fitoquímico, con predominio de polifenoles y derivados, compuestos fenólicos, flavonoides, taninos y antraquinonas (Figura 3).

Figura 3 - Análisis fitoquímico del extracto etanólico - ExtEtOH (200 mg mL $\left.\mathrm{mL}^{-1}\right)$, de las hojas de guaicuru caá (Cienfuegosia drummondii), Porto Murtinho, Mato Grosso do Sul, Brasil

\section{Clases de Metabolitos Secundarios}

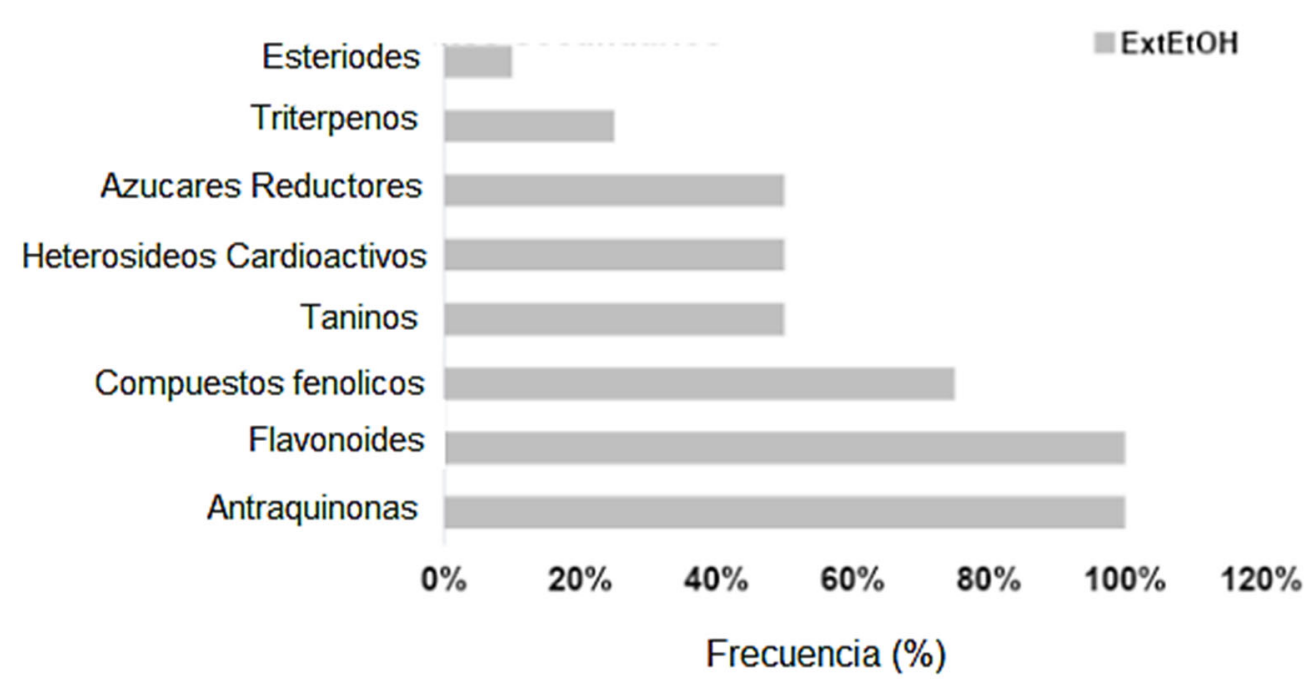

Fuente: Autoras.

De estas clases, los compuestos fenólicos, flavonoides y taninos fueron descritos para Cienfuegosia digitata Cav., una de las especies del género con mayor diversidad de estudios, pero para esta planta se mencionaron flavonoides, alcaloides, saponinas (KONATÉ et al., 2012a; VENKATESAN et al., 2019), y los polifenoles incluyen el gosipol que se encuentra en las raíces de las plantas (BOUNE et al., 2017), una sustancia que también se encuentra en los tallos, hojas, semillas y raíces de plantas pertenecientes al género Gossypium (GADELHA et al., 2011).

La alta frecuencia de taninos apoya la aplicación y forma de uso tópico por parte de la comunidad para el tratamiento y curación de heridas en la piel. Según informes de la población local, la planta de guaicuru caá también se utiliza para lavar heridas. Planta con alto contenido en taninos confiere acción cicatrizante e inhibidora sobre los microorganismos (NASCIMENTO; JESUS; ALVIM, 2021). Un estudio realizado por Martínez (2011) con comunidades indígenas del Chaco argentino señaló el uso de guaicuru caá para tratar síntomas gastrointestinales, posiblemente causados por microorganismos patógenos transportados por el agua. Scarpa y Anconatani (2017) también encontraron en registros históricos etnobotánicos de la provincia de Formosa, en el Chaco argentino, el uso de esta planta, en decocción de hojas, para tratamiento antisifilítico, además del tratamiento de heridas cutáneas. También en Argentina, Krapovickas (2003) encontró informes sobre el uso místico de esta planta por parte de las poblaciones locales.

También se analizaron los contenidos de compuestos fenólicos y flavonoides y otros aspectos fisicoquímicos (Cuadro 1). 
Cuadro 1 - Contenido de compuestos fenólicos y flavonoides del extracto etanólico, $\mathrm{pH}$ y conductividad eléctrica de guaicuru caá (Cienfuegosia drummondii), Porto Murtinho, Mato Grosso do Sul, Brasil

\begin{tabular}{|c|c|c|c|}
\hline $\begin{array}{c}\text { Compuestos fenólicos } \\
\mathbf{m g ~ g}^{-1} \text { de ácido gálico }\end{array}$ & $\begin{array}{c}\text { Flavonoides } \\
\mathbf{m g ~ g}^{-1} \text { de quercetina }\end{array}$ & $\mathbf{p H}$ & $\begin{array}{c}\text { Conductividad electrica } \\
(\boldsymbol{\mu S} / \mathbf{c m})\end{array}$ \\
\hline $101,66 \pm 0,01$ & $139,04 \pm 0,02$ & $5,19 \pm 0,0$ & $16,54 \pm 0,1$ \\
\hline
\end{tabular}

Fuente: Autoras.

Los niveles de compuestos fenólicos obtenidos para las hojas de C. drummondii fueron superiores a los encontrados por Konaté et al. (2012a) para especímenes de C. digitata, cuyo contenido estuvo entre $23,60 \pm 0,03$ y 61,23 $\pm 1,2 \mathrm{mg} \mathrm{GAE} / \mathrm{g}$. Los contenidos de flavonoides siguieron el mismo perfil, fueron superiores a la especie $C$. digitalis, con valores entre $3,83 \pm$ 0,07 y 10,83 $\pm 0,02 \mathrm{mgQE} / \mathrm{g}$. Los autores, incluso encontrando valores inferiores a los obtenidos para la planta en estudio, como se muestra en la Tabla (1), señalaron el efecto antioxidante de la planta sobre estas clases de fitoconstituyentes. Con base en esta información, es posible inferir que el extracto etanólico de $C$. drummondii tiene potencial antioxidante, lo que puede justificar los efectos terapéuticos atribuidos a la planta por las comunidades del Chaco.

Los flavonoides representan uno de los grupos fenólicos más importantes y diversos entre los productos de origen natural, con varias actividades biológicas atribuidas a esta clase de polifenoles, como antitumorales, antioxidantes, antivirales y antiinflamatorios (COUTINHO; MUZITANO; COSTA, 2009). Los compuestos fenólicos son plenamente reconocidos por sus propiedades antioxidantes (SHAHIDI; WANASUNDARA, 1992).

La presencia de polifenoles puede estar asociada con el efecto terapéutico confirmado para especies del género Cienfuegosia, como la hepatoprotección del extracto acuoso de C. digitata en ratas albinas (KONATÉ et al., 2012b). A los alcaloides, no detectados en el extracto etanólico de $C$. drummondii, se les atribuyó el potencial antimicrobiano frente a Staphylococcus aureus resistente a meticilina (MRSA) para $C$. digitata, conformando las características de esta planta como fuente de agente antibacteriano que puede ser utilizado eficazmente. para fines futuros de salud (KONATÉ et al., 2012a).

Además de los flavonoides, las antraquinonas también mostraron una frecuencia del 100\%. Es necesario profundizar en los estudios de actividad fitoquímica y biológica de esta especie para determinar, por ejemplo, los tipos de antraquinonas, si están disponibles durante todo el año o si la producción es estacional. Algunas plantas producen toxoides que pueden actuar como laxantes, mientras se utilizan en la terapia del cáncer de ovario (GOBBO-NETO; LOPES, 2007). Sin embargo, Arcanjo et al. (2013) advierten sobre el peligro del embarazo humano al utilizar plantas con presencia de antraquinonas, ya que es un laxante antranoide, que tiene como principio activo los sinusidos A y B farmacológicamente inactivos, comportándose como fármacos pronaturales.

Gossypium barbadense, algodón, que pertenece a la misma familia botánica que $C$. drummondii (Malvaceae), tiene propiedades abortivas en la raíz (POTT; POTT, 1994). Arenas y Azorero (1977) informaron que los indígenas de la etnia Chamacoco, residentes en Paraguay, utilizaban el decocto (hervir en agua) de la raíz de guaicuru caá, o la raíz macerada en mate, como método anticonceptivo, usándolo todos los meses, durante varios días.

En cuanto al pH obtenido del extracto etanólico, con tendencia a la acidez, y la conductividad eléctrica, estos son datos importantes para las plantas con potencial para obtener una fitoterapia 
tópica u oral. Según Simões et al. (2010), la acidez está relacionada con ácidos orgánicos, como compuestos fenólicos ácidos, taninos y saponinas.

La conductividad eléctrica es un parámetro físico importante para la producción de fitomedicinas y está relacionada con la concentración de electrolitos en solución, sólidos en suspensión, fuerza electrolítica y presencia de estructuras celulares (MIN; SASTRY; BALASUBRAMANIAM, 2007). Para Lewicki (2004), la conductividad eléctrica ocurre en medios que contienen moléculas cargadas eléctricamente positiva y negativamente, es decir, el resultado de la suma de todos los iones presentes en solución. En este estudio, la conductividad eléctrica fue baja, lo que permite inferir que los componentes químicos no están ionizados o es posible que exista una barrera por taninos y saponinas en la migración de iones al electrodo. Para esta hipótesis, estudios con otros disolventes hidrófilos corroborarán la presencia o ausencia de grupos ionizables en las clases de metabolitos secundarios.

Las experiencias familiares de la autora MB, así como los diálogos y experiencias confirmaron que la planta es ampliamente utilizada como medicina herbal en Porto Murtinho y las ciudades paraguayas aledañas. Si bien no se realizaron entrevistas estructuradas con vecinos de Porto Murtinho, varios informes confirmaron el uso medicinal popular del guaicuru caá (ingestión y uso tópico) para el tratamiento, prevención y combate de diversas enfermedades, especialmente antitumorales y antiinflamatorias. Y existe congruencia entre usos en toda la población del Chaco (Cuadro 2).

Cuadro 2 - Usos, ubicación y formas de preparación del guaicuru caá por comunidades del Chaco Sudamericano

\begin{tabular}{|c|c|c|c|}
\hline Usos & Localidad - Comunidad & Forma de uso/preparación & Referencia \\
\hline Método anticonceptivo & $\begin{array}{l}\text { Chaco Paraguayo- Comunidad } \\
\text { indígena Chamacoco y Lengua }\end{array}$ & $\begin{array}{l}\text { Decocción de las raíces, se } \\
\text { bebe en el mate (bebida) }\end{array}$ & $\begin{array}{l}\text { Arenas e } \\
\text { Azorero } \\
\text { (1977) }\end{array}$ \\
\hline $\begin{array}{l}\text { Dolor estomacal } \\
\text { (tratamiento } \\
\text { gastrointestinal) }\end{array}$ & $\begin{array}{l}\text { Chaco Argentino (provincia de } \\
\text { Chaco)- Comunidad indígena } \\
\text { Los Tobas (Qom) }\end{array}$ & $\begin{array}{l}\text { Decocción de las hojas en } \\
\text { agua, la infusión se bebe en } \\
\text { mate o terere (bebida) }\end{array}$ & $\begin{array}{l}\text { Martínez } \\
(2011)\end{array}$ \\
\hline Tratamiento antisifilítico & $\begin{array}{l}\text { Chaco Central Argentina } \\
\text { (provincia de Formosa)- } \\
\text { Comunidad indígena Los Qom }\end{array}$ & \begin{tabular}{|l|} 
Decocción de las hojas en \\
agua, se bebe en tereré \\
(bebida) y aplicación tópica
\end{tabular} & $\begin{array}{l}\text { Scarpa e } \\
\text { Anconatani } \\
\text { (2017) }\end{array}$ \\
\hline $\begin{array}{l}\text { Tratamiento de heridas } \\
\text { cutáneas }\end{array}$ & $\begin{array}{l}\text { Chaco Central Argentina } \\
\text { (provincia de Formosa)- } \\
\text { Comunidad indígena Los Qom }\end{array}$ & $\begin{array}{l}\text { Aplicación tópica de la } \\
\text { decocción de las hojas }\end{array}$ & $\begin{array}{l}\text { Scarpa e } \\
\text { Anconatani } \\
(2017)\end{array}$ \\
\hline Místico & $\begin{array}{l}\text { Chaco Central Argentina } \\
\text { (provincia de Formosa) - } \\
\text { Comunidad de Las Lomitas }\end{array}$ & $\begin{array}{l}\text { Mezcla de tabaco con } \\
\text { algodón de las semillas, se } \\
\text { fuma }\end{array}$ & $\begin{array}{l}\text { Krapovickas } \\
(2003)\end{array}$ \\
\hline $\begin{array}{l}\text { Analgésico, tratamiento } \\
\text { y combate a tumores } \\
\text { diversos, problemas } \\
\text { gástricos y uterinos, } \\
\text { depurativo de la sangre } \\
\text { y uso tópico (lavar) } \\
\text { para tratamiento de } \\
\text { heridas cutáneas (de la } \\
\text { piel, incluido el cuero } \\
\text { cabelludo) }\end{array}$ & $\begin{array}{l}\text { Chaco brasilero- Comunidad } \\
\text { de Porto Murtinho }\end{array}$ & $\begin{array}{l}\text { Decocción de hojas } \\
\text { e ingesta de té, uso } \\
\text { tópico(lavar), hojas } \\
\text { silvestres maceradas para el } \\
\text { mate o terere. }\end{array}$ & Este estudio \\
\hline
\end{tabular}

Fuente: Autoras. 
Los usos reportados por los residentes de Porto Murtinho son compartidos por los pueblos del Chaco, como se muestra en la tabla. Aunque los estudios sobre plantas medicinales han aumentado, aún son escasos en comparación con las dimensiones territoriales de Brasil y su diversidad vegetal (GUARIM-NETO; MORAIS, 2003; OLIVEIRA et al., 2009) y diversidad cultural.

Guarim-Neto y Morais (2003) señalan que se debe fomentar la investigación en Brasil con plantas medicinales, ya que es considerado el país biológicamente más rico del mundo y los estudios podrían conducir a la reorganización de estructuras para el uso de bienes naturales y un aumento del PIB per cápita, teniendo en cuenta que existe una gran tendencia mundial al aumento del uso de medicamentos a base de plantas.

Todavía hay una falta de estudios fitoquímicos y farmacológicos de plantas del Chaco brasileño, sobre todo porque muchas son comúnmente utilizadas como hierbas medicinales por la comunidad. Y, también, útil en alimentación (BORTOLOTTO et al., 2019). Tales usos adquieren singularidad en sus prácticas culturales, dado que en esta región geográfica confluyen diversas culturas que se integran en un territorio de alta riqueza étnica y expresividad, especialmente de pueblos originarios del Chaco. Ferreira, Ilho y Oliveira (2019) destacan que los habitantes del Chaco presentan un verdadero caleidoscopio cultural, resultado de la ocupación histórica de diferentes etnias indígenas.

Se da una fuerte integración cultural entre brasileños y paraguayos en esta región fronteriza, incluyendo el uso de plantas medicinales. Como describen Benites et al. (2019), mujeres paraguayas de Carmelo Peralta y otras comunidades a lo largo del río Paraguay, por lo tanto, la vecina Porto Murtinho, a menudo cruzan el río trayendo plantas medicinales para la venta en la feria de Porto Murtinho, práctica que confirma la posesión de conocimientos tradicionales, el intercambio del conocimiento etnobiológico y la integración de los pueblos de un mismo territorio espacial.

El uso medicinal de las plantas es solo una de las interfaces de la etnobotánica, y se necesitan más estudios que involucren otras relaciones, propósitos y formas de uso de las plantas nativas por parte de las comunidades locales.

La aproximación entre ciencia académica y conocimiento tradicional propone la apertura de nuevas perspectivas de desarrollo, capaces de valorar prácticas culturales, muchas de las cuales se remontan a generaciones y saberes de pueblos originarios. Mantener vivo el patrimonio cultural y natural son requisitos previos esenciales para la sostenibilidad y, por tanto, para la comprensión de la sociedad y el desarrollo local. Y cabe señalar que, sin una biodiversidad conservada, todos estos patrimonios sociohistóricos corren el riesgo de ser olvidados, suplantados o incluso desaparecer a largo plazo, ya que las condiciones materiales se imponen en la sociedad del consumo. Porto Murtinho Chaco es la región más afectada por la deforestación en la llanura del Pantanal (SILVA et al., 2008).

La forma de uso de los bienes naturales, como las plantas en la medicina popular, amplía la relación afectiva con la naturaleza, que se refleja en las relaciones sociales, y viceversa. Pardode-Santayana y Macía (2015) creen que los valores culturales y simbólicos, basados en el uso de plantas nativas con fines alimentarios o medicinales, tienen un gran impacto en los conocimientos tradicionales y en las relaciones sociales de los grupos étnicos; y el uso sostenible favorece la conservación de la sociobiodiversidad.

Por lo tanto, este estudio documenta el uso del guaicuru caá por parte de la comunidad chaqueña brasileña y destaca la fuerte relación en prácticas culturales de las comunidades del 
Chaco sudamericano con su territorio y las plantas, además de brindar informaciones relevantes para propuestas de desarrollo que priorizan la sustentabilidad.

\section{CONSIDERACIONES FINALES}

Este estudio proporciona información básica de diagnóstico sobre las propiedades fitoquímicas de una planta utilizada en la frontera entre Brasil y Paraguay, en Porto Murtinho. Como señalaron los resultados, la determinación de componentes bioactivos en plantas ofrece respaldo científico para el uso medicinal popular.

Este entrelazamiento de conocimientos puede traer beneficios mutuos y contribuir a la conservación a largo plazo de la biodiversidad, a la satisfacción de las necesidades humanas y a la producción de medios de vida en el contexto histórico-social. Duarte (2008) afirma que un objeto natural para ser apropiado y convertirse en instrumento, es decir, ligado a su utilidad práctica para el ser humano, requiere el conocimiento de sus propiedades naturales.

Así, los compuestos fitoquímicos, aquí presentados, dan cuenta de parte del conocimiento requerido para la incorporación de la práctica cultural, es decir, el uso medicinal del guaicuru caá como práctica social en las comunidades del Chaco sudamericano.

Es posible ver cuán ricas son las prácticas culturales de diferentes pueblos en su relación con la naturaleza y su apropiación social, lo que reafirma su importancia e irremplazabilidad para todas las culturas. Además, destacamos la relación de las poblaciones con la biodiversidad en la frontera brasileña con Paraguay y su potencial contribución al turismo a ser promovido en la Ruta Bioceánica, que demanda políticas públicas para el mejoramiento de las relaciones con la sociobiodiversidad con participación real de las comunidades en la toma de decisiones.

De una mirada cercana a los territorios que atraviesa la Ruta Bioceánica, se puede apreciar la pluralidad de saberes, pueblos, etnias, culturas, tradiciones y saberes que se transmiten de generación en generación. La Ruta, que consta de más de dos mil kilómetros en un recorrido lineal, puede contribuir a la integración del conocimiento latinoamericano, que demanda visiones no lineales del territorio y las comunidades. La transformación radical de los paisajes chaqueños pone en riesgo la biodiversidad y su uso social, por ende, la sociobiodiversidad.

En la expectativa de integración cultural y reducción de fronteras, la valorización y protagonismo de diferentes prácticas culturales no hegemónicas, como las vinculadas a la etnobotánica y la etnociencia general, pueden atribuir un valor superlativo al turismo cultural en la ansiada Ruta de la Integración Latinoamericana.

\section{AGRADECIMIENTOS}

Queremos agradecer a Uniderp-Anhanguera y al Laboratorio de Morfología Vegetal por su ayuda en la realización de los análisis fitoquímicos. Agradecemos a Vivian A. Assunção por sus valiosas sugerencias sobre el manuscrito. MB agradece el apoyo de la Universidade Federal de Mato Grosso do Sul- UFMS / MEC- Brasil y la Coordinación para el Perfeccionamiento del Personal de Educación Superior- Brasil (CAPES) por otorgar una beca- Código de Financiamiento 001. 


\section{REFERENCIAS}

ALBUQUERQUE, U. P.; RAMOS, M. A.; FERREIRA-JUNIOR, W. S.; MEDEIROS, P. M. Ethnobotany for beginners. Suiça: Switzerland: Springer Nature, 2017.

ARCANJO, G. M. G.; MEDEIROS, M. L. F. S.; AZEVEDO, R. R. S.; GRIZ, S. A. S.; ROCHA, T. J. M.; MOUSINHO, K. C. Estudo da utilização de plantas medicinais com finalidade abortiva. Revista Eletrônica de Biologia, v. 6, n. 3, p. 234-250, 2013.

ARENAS, P.; AZORERO, M. Plants used as means of abortion, contraception, sterilization and fecundation by Paraguayan indigenous people. Economic Botany, New York, v. 31. n. 3, p. 302-6, 1977.

BENITES, M.; MAMEDE, S.; CENTENO, C. V.; ALVES, G. L. Porto Murtinho, Mato Grosso do Sul e a Rota Bioceânica: caminhos possíveis para sustentabilidade socioambiental. Interações, Campo Grande, MS, v. 20, n. especial, p. 267-84, 2019.

BORTOLOTTO, I. M.; SELEME, E. P.; ARAÚJO, I. P. P.; MOURA, S. S.; SARTORI, A. L. B. Conhecimento local sobre plantas alimentícias nativas no Chaco brasileiro. Oecologia Australis, Rio de Janeiro, v. 23, n. 4, p. 764-75, 2019.

BOUNE, M. V. S. et al. Development of SECheM concept for isolation and chemical modification of gossypol directly from Cienfuegosia digitata. Phytochemical Analysis, [s.I.], v. 28, n. 5, p, 410-5, 2017.

CARVALHO, F. S.; SARTORI, A. L. B. Reproductive phenology and seed dispersal syndromes of woody species in the Brazilian Chaco. Journal of Vegetation Science, [s.I.], v. 26, n. 2, p. 302-11, 2014.

CORDEIRO, J. M. P.; FÉLIX, L. P. Conhecimento botânico medicinal sobre espécies vegetais nativas da Caatinga e plantas espontâneas no agreste da Paraíba, Brasil. Revista Brasileira de Plantas Medicinais, Campinas, v. 16, n. 3 (supl. I), p. 685-92, 2014.

COUTINHO, M. A.; MUZITANO, M. F.; COSTA, S. S. Flavonoides: potenciais agentes terapêuticos para o processo inflamatório. Revista Virtual de Química, Niterói, v. 1., n. 3., p. 241-256, 2009.

DUARTE, N. Sociedade do conhecimento ou sociedade das ilusões?. Campinas: Autores associados, 2008.

FERREIRA, M. L.; CASTILHO, M. A.; OLIVEIRA, E. M. Brasil, Paraguai, Argentina e Chile/Rota Bioceânica: relações culturais no território vivido. Interações, Campo Grande, v. 20, n. especial, p. 69-89, 2019.

FONTOURA, F. M.; MATIAS, R.; LUDWIG, J.; OLIVEIRA, A. K. M. D.; BONO, J. A. M.; MARTINS, P. F. R. B.; CORSINO, J.; GUEDES, N. M. R. Seasonal effects and antifungal activity from bark chemical constituents of Sterculia apetala (Malvaceae) at Pantanal of Miranda, Mato Grosso do Sul, Brazil. Acta Amazonica, Manaus, v. 45., n. 3, p. 283-292, 2015.

FRANCO, E. A. P.; BARROS, R. F. M. Uso e diversidade de plantas medicinais no Quilombo Olho D'água dos Pires, Esperantina, Piauí. Revista Brasileira de Plantas Medicinais, Campinas, v. 8., n. 3, p. 78-88, 2006.

FREITAS, T. G.; SOUZA, C. S.; AOKI, C.; ARAKAKI, L. M. M.; STEFANELLO, T. H.; SARTORI, A. L. B.; SIGRIST, M. R. Flora of Brazilian humid Chaco: composition and reproductive phenology. Check List, [s.I.], v. 9, n. 5, p. 973-9, 2013.

FRYXELL, P. A. The interpretation of disjunct distributions. Taxon, [s.I.], v. 16., n. 4, p. 316-24, 1967.

GADELHA, I. C. N.; RANGEL, A. H.; SILVA, A. R.; SOTO-BLANCO, B. Efeitos do gossipol na reprodução animal. Acta Veterinaria Brasilica, Mossoró, v. 5, n. 2, p. 129-35, 2011. 
GIRALDI, M.; HANAZAKI, N. Uso e conhecimento tradicional de plantas medicinais no Sertão do Ribeirão, Florianópolis, SC, Brasil. Acta Botanica Brasilica, Brasília, v. 24., n. 2, p. 395-406, 2010.

GOBBO-NETO, L.; LOPES, N. P. Plantas medicinais: fatores de influência no conteúdo de metabólitos secundários. Química nova, São Paulo, v. 30., n. 2, p. 374-81, 2007.

GUARIM-NETO, G.; MORAIS, R. G. Recursos medicinais de espécies do Cerrado de Mato Grosso: um estudo bibliográfico. Acta Botanica Brasilica, Brasília, v. 17., n. 4, p. 561-584, 2003.

JESUS, N. Z. T. D.; LIMA, J. C. D. S.; SILVA, R. M. D.; ESPINOSA, M. M.; MARTINS, D. T. D. O. Levantamento etnobotânico de plantas popularmente utilizadas como antiúlceras e antiinflamatórias pela comunidade de Pirizal, Nossa Senhora do Livramento-MT, Brasil. Revista Brasileira de Farmacognosia, Seropédica, v. 19, n. 1, p. 130-39, 2009.

JONES, R. W. Evolution of the host plant associations of the Anthonomus grandis species group (Coleoptera: Curculionidae): phylogenetic tests of various hypotheses. Annals of the Entomological Society of America, [s.I.], v. 94, n. 1, p. 51-8, 2001.

KONATÉ, K.; OUÉdRAOGO, M.; OUATTARA, M. B.; DIBALA, I. C.; MAVOUNGOU, J. F.; LEPENGUE, A. N., SOUZA, A.; BATCHI, B. M.; NACOULMA, O. G. Hepatoprotective activity of aqueous acetone extract from Cienfuegosia digitata Cav. (Malvaceae) against alcohol hepatotoxicity in albinos Wistar Rats. Research Journal of Applied Sciences, Engineering and Technology, [s.I.], v. 4., n. 12, p. 1637-41, 2012a.

KONATÉ, K.; MAVOUNGOU, J. F.; LEPENGUÉ, A. N.; AWORET-SAMSENY, R. R.; HILOU, A.; SOUZA, A.; MAMOUDOU, H. D.; M'BATCHI, B. Antibacterial activity against $\beta$-lactamase producing Methicillin and Ampicillin-resistants Staphylococcus aureus: fractional Inhibitory Concentration Index (FICI) determination. Annals of clinical microbiology and antimicrobials, [s.I.], v. 11, n. 1, p. 1-12, 2012 b.

KRAPOVICKAS, A. Las species Austroamericanas del género Cienfuegosia CAV. (Malvaceae-Gossypieae). Bonplandia, Corrientes, v. 12, n. 1-4, p. 5-47, 2003.

KUMAR, D.; KUMAR, A.; PRAKASH, Potential antifertility agents from plants: a comprehensive review. Journal of Ethnopharmacology, [s.I.], v. 140, n. 1, p. 1-32, 2012.

LANTERI, A. A.; CONFALONIERI, V. A.; SCATAGLINI, M. A. El picudo del algodonero en la Argentina: principales resultados e implicancias de los estudios moleculares. Revista de la Sociedad Entomológica Argentina, [s.I.], v. 62, n. 3-4, p. 1-15, 2003.

LEFF, E. Ecologia, capital e cultura: a territorialização da racionalidade ambiental. Petrópolis: Vozes, 2009.

LEWICKI, P. P. Water as the determinant of food engineering properties. Food Engineering Review, [s.l.], v. 61, p. 483-95, 2004.

LEWTON, F. L. Cienfuegosia drummondii, a rare Texas plant. Bulletin of the Torrey Botanical Club, [s.I.], v. 37, n. 9, p. 473-75, 1910.

LIMA, R. A.; MAGALHÃES, S. A.; SANTOS, M. R. A. Levantamento etnobotânico de plantas medicinais utilizadas na cidade de Vilhena, Rondônia. Revista Pesquisa \& Criação, Porto Velho, v. 10, n. 2, p. 165179, 2011.

MACIEL, M. A. M.; PINTO, A. C.; VEIGA-JUNIOR, V. F. Plantas medicinais: a necessidade de estudos multidisciplinares. Química Nova, São Paulo, v. 25, n. 3, p. 429-38, 2002. 
MARTINELLI, G.; MORAES, M. A. Livro vermelho da flora do Brasil. Rio de Janeiro: Andrea Jakobsson; Instituto de Pesquisas Jardim Botânico do Rio de Janeiro, 2013.

MARTíNEZ, G. J. Use of medicinal plants in the treatment of waterborne diseases in a Toba (qom) community of the "Impenetrable" (Chaco, Argentina): an ethnoecological and sanitary perspective. Bonplandia, Corrientes, AR, v. 20, n. 2, p. 329-52, 2011.

MATOS, J. F. A. Introdução a fitoquímica experimental. Fortaleza: UFC. 2009

MERELES, M. F. Una aproximación al conocimiento de las formaciones vegetales del Chaco Boreal, Paraguay. Rojasiana, Asunción, v. 6, n. 2, p. 5-48, 2005.

MESSIAS, M. C. T. B.; MENEGATTO, M. F.; PRADO, A. C. C.; SANTOS, B. R.; GUIMARÃES, M. F. M. Uso popular de plantas medicinais e perfil socioeconômico dos usuários: um estudo em área urbana em Ouro Preto, MG, Brasil. Revista Brasileira de Plantas Medicinais, São Paulo, v. 17, n. 1, p. 76-104, 2015.

MIN, S.; SASTRY, S. K.; BALASUBRAMANIAM, V. M. In situ electrical conductivity measurement of select liquid foods under hydrostatic pressure to 800 MPa. Journal of food engineering, [s.I.], v. 82, n.4, p. 489-97, 2007.

MOREIRA, R. D. C. T.; COSTA, L. C. D. B.; COSTA, R. C. S.; ROCHA, E. A. Abordagem etnobotânica acerca do uso de plantas medicinais na Vila Cachoeira, Ilhéus, Bahia, Brasil. Acta farmacêutica bonaerense, Buenos Aires, v. 21, n. 3, p. 205-11, 2002.

MOURA, S. S. Quintais como espaços para conservação no Chaco. 2016. 73f. Dissertação (Mestrado em Biologia Vegetal) - Universidade Federal de Mato Grosso do Sul, Campo Grande, MS, 2016.

NASCIMENTO, I. J. R.; JESUS, H. S.; ALVIM, H. G. O. Uso dos taninos provenientes do barbatimão para cicatrização de ferimentos. Revista JRG de Estudos Acadêmicos, Brasília, v. 4, n. 8, p. 201-12, 2021.

OLIVEIRA, F. C. D.; ALBUQUERQUE, U. P. D.; FONSECA-KRUEL, V. S. D.; HANAZAKI, N. Avanços nas pesquisas etnobotânicas no Brasil. Acta Botanica Brasilica, Brasília, v. 23, n. 2, p. 590-605, 2009.

PANDEY, A. K.; TRIPATHI, Y. C. Ethnobotany and its relevance in contemporary research. Journal of Medicinal Plants Studies, Delhi, v. 5, n. 3, p. 123-29, 2017.

PARDO-DE-SANTAYANA, M; MACÍA, M. J. The benefits of traditional knowledge. Nature, London, v. 518, 487-8, 2015.

PEIXOTO-SOBRINHO, T. J. S.; SILVA, C. H. T. P.; NASCIMENTO, J. E.; MONTEIRO, J. M.; ALBUQUERQUE, U.P. AMORIM, E. L. C.; Validação de metodologia espectrofotométrica para quantificação dos flavonóides de Bauhinia cheilantha (Bongard) Steudel. Revista Brasileira de Ciências Farmacêuticas, São Paulo, v. 44, p. 683-9, 2008.

PENNINGTON, R. T.; PRADO, D. E.; PENDRY, C. A. Neotropical seasonally dry forests and quarternary vegetation changes. Journal of Biogeography, Los Angeles, v. 27, n. 2, p. 261-73, 2000.

POTT, A.; POTT, V. J. Plantas do Pantanal. Corumbá: Embrapa-SPI, 1994.

PRADO, D. E. What is the Gran Chaco vegetation in South America? A redefinition. Contribution to the study of flora and vegetation of the Chaco. VII. Candollea, v. 48, n. 2, p. 615-629, 1993.

RIBEIRO, D. A.; MACÊDO, D. G.; OLIVEIRA, L. G. S.; SARAIVA, M. E.; OLIVEIRA, S. F.; SOUZA, M. M. A.; MENEZES, I. R. A. Potencial terapêutico e uso de plantas medicinais em uma área de Caatinga no estado do Ceará, nordeste do Brasil. Revista Brasileira de Plantas Medicinais, Campinas, v. 16, n. 4, p. 912-30, 2014. 
RODRIGUES, V. E. G.; CARVALHO, D. D. Levantamento etnobotânico de plantas medicinais no domínio do cerrado na região do Alto Rio Grande-Minas Gerais. Ciência e Agrotecnologia, Lavras, v. 25, n. 1, p. 102-23, 2001.

SALOMÃO, A. K. D.; POTT, A.; SARTORI, A. L. B.; ASSUNÇÃO, V. A. Espécies herbáceo-arbustivas do Chaco brasileiro e uso potencial. Revista Brasileira de Agroecologia, Recife, v. 4, n. 2, p. 2238-41, 2009.

SARTORI, A. L. B. The Brazilian Chaco. Glalia, v. 4, n. 1, p. 11-18, 2012.

SCARPA, G. F.; ANCONATANI, L. M. Etnobotánica histórica de las misiones franciscanas del este de Formosa II: identificación y análisis de datos inéditos y reelaboración integral de fuentes ya publicadas a partir de hallazgos documentales. Dominguezia, [s.I.], v. 33, n. 2, p. 37-79, 2017.

SCHMID, K. M.; PATTERSON, G. W. Distribution of cyclopropenoid fatty acids in malvaceous plant parts. Phytochemistry, [s.l.], v. 27, n. 9, p. 2831-34, 1988.

SHAHIDI, F.; JANITHA, P. K.; WANASUNDARA, P. D. Phenolic antioxidants. Critical Reviews in food Science \& Nutrition, [s.I.], v. 32. n. 1, p. 67-103, 1992.

SISTEMA DE INFORMAÇÃO SOBRE A BIODIVERSIDADE BRASILEIRA [SIBBr]. Lista de Ameaça de Flora e Fauna do Estado do Rio Grande do Sul - avaliação de 2014. SIBBr. [s.l], 2021. Disponible en: https:// specieslist.sibbr.gov.br/speciesListltem/list/drt1572898055708?q=DRUMMONDII. Acceso el: 2 mayo 2021.

SILVA, M. P.; MAURO, R. A.; ABDON, M.; SILVA, J. S. V. Estado de Conservação do Chaco (Savana Estépica) brasileiro. In: SIMPÓSIO NACIONAL CERRADO, 9.; SIMPÓSIO INTERNACIONAL SAVANAS TROPICAIS, 2., Brasília, 2-17 oct. 2008. Anales [...]. Brasília: Embrapa Cerrados, 2008. Disponible en: http://simposio. cpac.embrapa.br/simposio\%20em\%20pc210\%20(Pc210)/trabalhos_pdf/00789_trab1_ap.pdf. Acceso el: 2 marzo 2021.

SIMÕES, C. M. O. et al. Farmacognosia: da planta ao medicamento. 6. ed. Porto Alegre: Editora UFRGS; Florianópolis: Editora UFSC, 2010.

SOUSA, C. M.; SILVA, H. R. E.; VIEIRA-JR, G. M.; AYRES, M. C. C.; COSTA, C. L. S.; ARAÚJO, D. S. Fenóis totais e atividade antioxidante de cinco plantas medicinais. Química Nova, [s.I.], v. 30. p. 351-5, 2007.

THOMPSON, A. C.; HANNY, B. W.; HEDIN, P. A.; GUELDNER, R. C. Phytochemical studies in the family Malvaceae. I. Comparison of essential oils of six species by gas-liquid chromatography. American Journal of Botany, Hartford, v, 58, n. 9, p. 803-7, 1971.

VENKATESAN, G. K.; KUPPUSAMY, A.; DEVARAJAN, S.; KUMAR, A. K. K. Review on medicinal potential of alkaloids and saponins. Pharamacologyonline, [s.I.], v. 1, p. 1-20, 2019.

VOGT, C. Composición de la Flora Vascular del Chaco Boreal, Paraguay III. Dycotiledoneae: GesneriaceaeZygophyllaceae. Steviana, Asunción, v. 5, p. 5-40, 2013.

WORKMAN-JUNIOR, J. J. Infrared and Raman spectroscopy in paper and pulp analysis. Applied Spectroscopy Reviews, [s.I.], v. 36, n. 2-3, p. 139-68, 2001. 


\section{Sobre las autoras:}

Maristela Benites: Estudiante de Doctorado en Enseñanza de Ciencias en la Universidad Federal de Mato Grosso do Sul (UFMS), Brasil. Magíster en Ecología y Conservación, y Licenciada en Ciencias Biológicas por la UFMS. Investigadora y educadora ambiental del Instituto Mamede de Investigaciones Ambientales y Ecoturismo. E-mail: maris.benites@gmail.com, Orcid: http://orcid.org/0000-0003-0024-1122

Simone Mamede: Doctora y Magíster en Medio Ambiente y Desarrollo Regional por la Universidad para el Desarrollo del Estado y Región del Pantanal (Uniderp), Brasil. Especialista en Ecoturismo y Educación Ambiental por la Universidad Federal de Lavras (UFLA). Licenciada en Ciencias Biológicas por la Uniderp, Graduada en Gestión Turística por la Universidad Estácio de Sá, y Bachiller en Turismo por la Universidad do Sul de Santa Catarina (UNISUL). Socio-directora de la microempresa Instituto Mamede de Investigaciones Ambientales y Ecoturismo, Brasil. Trabaja como investigadora en Turismo y Biodiversidad en los diferentes biomas de Sudamérica. Profesora de curso de turismo en la UNESP, campus experimental de Rosana. E-mail: simone.mamede1@gmail.com, Orcid: http://orcid.org/0000-0002-7785-320X

Rosemary Matias: Doctora y Magíster en Química por la Universidad Estadual de Maringá (UEM), Brasil. Licenciada en Química por la Universidad Federal de Mato Grosso do Sul (UFMS). Profesora adjunta I de la Universidad Anhanguera-Uniderp. E-mail: rosematias@uniderp.edu.br, Orcid: https://orcid.org/0000-0002-0154-1015

Icléia Albuquerque de Vargas: Doctora en Medio Ambiente y Desarrollo por la Universidad Federal de Paraná (UFPR), Brasil, con prácticas de doctorado en LADYSS (Laboratoire Dynamiques Sociales et Recomposition des Espaces) / Paris X Nanterre. Magíster en Educación y Licenciado en Geografía por la Universidad Federal de Mato Grosso do Sul (UFMS). Profesora asociada de la Facultad de Arquitectura y Urbanismo, Ingeniería y Geografía (FAENG) de la UFMS. E-mail: icleiavargas12@gmail.com, Orcid: https://orcid.org/0000-0002-4214-4542 
